\title{
The Effect of Scattering on the Temperature Stratification of 3D Model Atmospheres of Metal-Poor Red Giants
}

\author{
Remo Collet $^{1}$, Wolfgang Hayek ${ }^{2,1,3}$, and Martin Asplund ${ }^{1}$ \\ ${ }^{1}$ Max Planck Institut für Astrophysik, Karl-Schwarzschild-Str. 1, D-85741 Garching, Germany \\ email: remo, hayek, asplund@mpa-garching.mpg.de \\ ${ }^{2}$ Research School of Astronomy \& Astrophysics, Cotter Road, Weston ACT 2611, Australia \\ ${ }^{3}$ Institute for Theoretical Astrophysics, Sem Sælands Vei, N-0315 Oslo, Norway
}

\begin{abstract}
We study the effects of different approximations of scattering in 3D radiationhydrodynamics simulations on the photospheric temperature stratification of metal-poor red giant stars. We find that assuming a Planckian source function and neglecting the contribution of scattering to extinction in optically thin layers provides a good approximation of the effects of coherent scattering on the photospheric temperature balance.
\end{abstract}

Keywords. stars: atmospheres, convection, hydrodynamics, radiative transfer, scattering, methods: numerical

\section{Introduction}

Three-dimensional model atmospheres of metal-poor late-type stars predict lower surface temperatures than their 1D counterparts. This leads to important differences between 3D and 1D spectroscopic analyses whenever temperature-sensitive features are used to determine elemental abundances (Asplund et al. 1999; Asplund \& García Pérez 2001; Collet et al. 2006, 2007). 3D models by various groups agree qualitatively, but not quantitatively on how cool the upper atmospheric layers in metal-poor stars actually are (Bonifacio et al. 2009; Bonifacio 2010). It has been suggested that such discrepancies and, in particular, the very low photospheric temperatures may be due to the approximated treatment of scattering in the radiative transfer. In this study, we explore the role of scattering on the predicted surface temperatures in 3D model atmospheres of metal-poor red giants.

\section{Method}

We use the 3D radiation-hydrodynamics code BIFROST (Gudiksen et al., in prep.) to construct surface convection simulations of a red giant star $\left(T_{\text {eff }}=5100 \mathrm{~K}, \log g\left[\mathrm{~g} \mathrm{~cm}^{-2}\right]=\right.$ $2.2,[\mathrm{Fe} / \mathrm{H}]=-3)$. We account for the effects of radiative cooling by solving the radiative transfer equation at each time-step. For the treatment of scattering, we consider three cases: (i) no contribution of continuum scattering to extinction in optically thin layers (Asplund et al. 1999; Collet et al. 2007); (ii) scattering treated as pure absorption everywhere; (iii) proper iterative solution of the radiative transfer equation in the case of a source function with coherent scattering term (Hayek et al. 2010). 


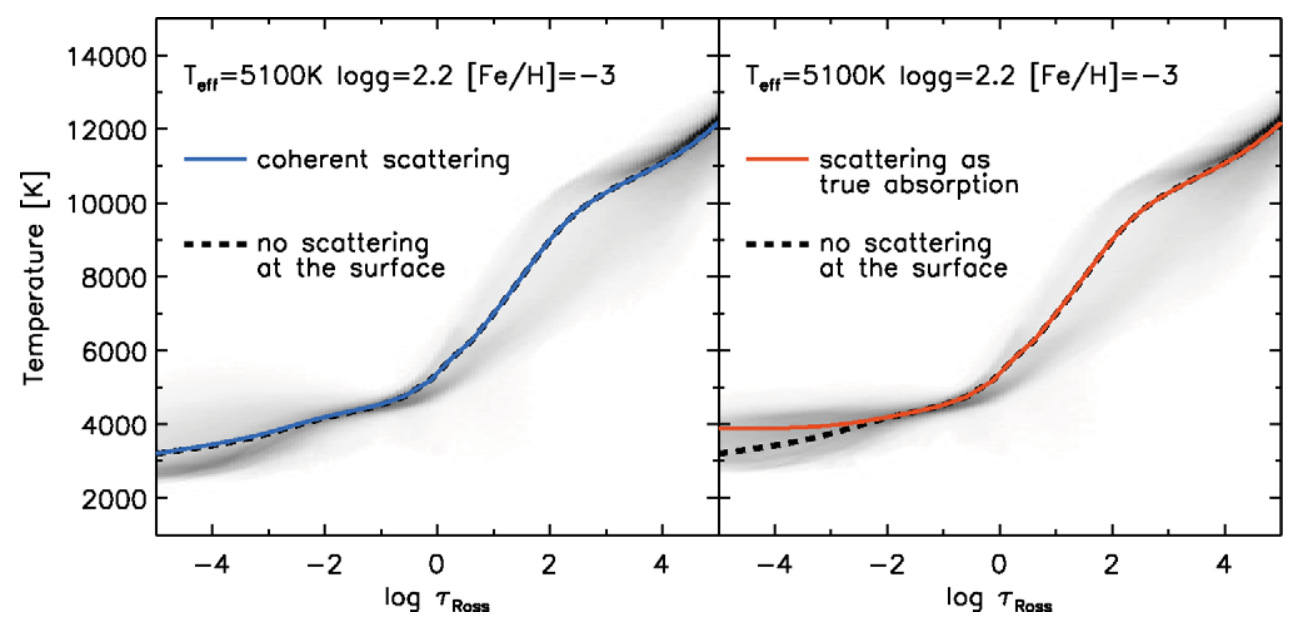

Figure 1. Left panel, grey shaded area: temperature distribution as a function of Rosseland optical depth in a snapshot of the 3D metal-poor red giant surface convection simulation computed for a source function with coherent scattering term. Continuous blue line: corresponding mean temperature stratification. Right panel, grey shaded area: temperature distribution in a snapshot of the 3D simulation assuming a Planckian source function and scattering as pure absorption. Dashed lines, both panels: mean temperature stratification for the simulation assuming a Planckian source function for the radiative transfer and no contribution of scattering to extinction in optically thin layers.

\section{Results}

The left panel of Fig. 1 shows the temperature distribution with optical depth in a 3D model metal-poor red giant atmosphere constructed by solving the radiative transfer equation in the case of a source function with coherent scattering term. The predicted temperature stratification is in very good agreement with the one obtained by simply assuming a Planckian source function and neglecting the contribution of scattering to the total extinction at the surface. Conversely, treating scattering as true absorption everywhere in the simulation leads to a significantly hotter temperature stratification.

To summarize, we have verified that, at least for 3D metal-poor red giant surface convection simulations, the assumption of a Planckian source function and of no scattering extinction in optically thin layers well reproduces the effects of coherent scattering on the photospheric temperature stratification. Consequently, we exclude that the cool temperature stratifications predicted by the $3 \mathrm{D}$ metal-poor red giant simulations of Collet et al. (2007) may be due to their approximated treatment of scattering.

\section{References}

Asplund, M., Nordlund, Å., Trampedach, R., \& Stein, R. F. 1999, A\&̛A, 346, L17

Asplund, M. \& García Pérez, A. E. 2001, A\&A, 372, 601

Bonifacio, P. 2010, in: K. Cunha, M. Spite, \& B. Barbuy (eds.), Chemical Abundances in the Universe: Connecting First Stars to Planets, Proc. IAU Symposium No. 265 (Rio de Janeiro), p. 81

Bonifacio, P., Spite, M., Cayrel, R., et al. 2009, A\& $A$, 501, 519

Collet, R., Asplund, M., \& Trampedach, R. 2006, ApJ (Letters), 644, L121

Collet, R., Asplund, M., \& Trampedach, R. 2007, A\& A, 469, 687

Hayek, W., Asplund, M., Carlsson, R., et al. 2010, A\&SA, in press 